\title{
ANALYSIS OF EEG RESPONSE FOR AUDIO-VISUAL STIMULI IN FRONTAL ELECTRODES AT THETA FREQUENCY BAND USING THE TOPOLOGICAL FEATURES
}

\author{
Himanshu Kumar1, \\ ${ }^{1}$ Department of Applied Mechanics, Indian Institute of Technology Madras, Chennai, India \\ ${ }^{2}$ Department of Electrical Engineering, National Institute of Technology Calicut, Kozhikode, India
}

Corresponding Author: Himanshu Kumar

Email: him241994@gmail.com

https://doi.org/10.34107/BiomedSciInstrum.57.04333

\begin{abstract}
Emotions are the fundamental intellectual capacity of humans characterized by perception, attention, and behavior. Emotions are characterized by psychophysiological expressions. Studies have been performed by analyzing Electroencephalogram (EEG) responses from various lobes of the brain under all frequency bands. In this work, the EEG response of the theta band in the frontal lobe is analyzed extracting topological features during audio-visual stimulation. This study is carried out using the EEG signals from the public domain database. In this method, the signals are projected in higher dimensional space to find out the geometrical properties. Features, namely the center of gravity and perimeter of the boundary space, are used to quantify the changes in the geometrical properties of the signal, and the features are subject to the Wilcoxon rank-sum test for statistical significance. Different electrodes in the frontal region under the same audio-visual stimulus showed similar variations in the geometry of the boundary in higher-dimensional space. Further, the electrodes, Fp1 and F3, showed a statistical significance of $\mathrm{p}<0.05$ in differentiating arousal states, and the Fp1 electrode showed a statistical significance in differentiating valence emotional state. Thus, the topological features extracted from the frontal electrodes in theta band could differentiate arousal and valence emotional states and be of significant clinical relevance.
\end{abstract}

Keywords: EEG, Emotion, Audio Visual Stimuli, Topological Features, Emotion recognition

\section{INTRODUCTION}

Electroencephalogram (EEG) is a clinical indicator of the brain-generated cortical neuronal electrical activity. It is recorded from the scalp's surface using multiple electrodes and is used for the analysis and diagnosis of various physiological, mental illness, and brain disorders [1-2]. One of the major causes of death worldwide is emotion-related mental disorder [3]. These emotional abnormalities are associated with depression, stress, and indecisive irritability. It can affect different aspects of human life, such as decision-making, perception, problem-solving, creativity, innovation, and effective social communication. Emotions also play a critical part in psychopathology and various psychological disorders like autism [4-5].

Several models have been reported to describe emotions. The Dimensional approach, namely, the Circumplex Model of Affect (CMA), is a widely preferred model to describe emotions. All the emotional states can be represented in two fundamental, independent, and orthogonal dimensions, namely arousal and valence using CMA. Valence is a measure of positive or negative affect towards an object, whereas arousal measures the intensity of the reaction towards an object: neutral or dominant [6].

Emotion recognition can be coarsely classified into two approaches, namely, non-physiological and physiological approaches [7]. Methods based on non-physiological signals include facial expressions, voices, and body gestures. However, the emotional responses obtained from these methods 